\title{
Ueber die Dipterengattung Alloeostylus Schnabl und über die sogenannten Kreuzborsten bei Anthomyiden- Weibchen.
}

\section{Von Jos. Mik in Wien.}

Nachdem ich mir einzig und allein im Interesse der Wissenschaft, also in der objectivsten Weise und fern von jedem persönlichen Gefühle, erlaubt hatte, den Vorgang des Herrn Dr. Schnabl: die mehr oder weniger berechtigten Anthomyiden-Gattungen Aricia, Spilogaster, Mydaea, Limnophora und Trichopticus (auctorum) in eine Gattung Aricia sens. lat. zusammenzuziehen, zu missbilligen (vgl. Entom. Nachr. 1887, pag. 234), hatte sich der Herr Doctor berufen gefunden, in einer nichts weniger als a nständigen Entgegnung (vgl. Entom. Nachr. 1887, pag. 343) gegen meine Ansichten zu eifern. Da verschiedene Entstellungen meiner Angaben die genannte Entgegnung auszeichnen, sich darin auch Invectiven gegen mich vorfinden, so war ich selbstverständlich gezwungen, eine "Antwort auf Herrn Dr. J. Schnabl's Entgegnung" (vgl. Entom. Nachr. 1888, pag. 41) zu geben, welche das rechte Licht dieser Entgegnung der Oeffentlichkeit zu vermitteln zum Zwecke hatte.

Nun kommt Herr Dr. Schnabl mit einer neuen Anthomyidengattung, genannt Alloeostylus sibi (vgl. Ent. Nachr. 1888, pag. 49), deren Charakteristik meine abfällige Kritik über das Genus Aricia sens. lat. (Schnabl) im vollsten Sinne des Wortes als berechtigt erklärt. Niemand könnte die in den ${ }_{n}$ Contributions à la Faune Diptérologique" von Herrn Dr. Schnabl gegebenen Auseinandersetzungen über Aricia s. l. schlagender ad absurdum führen, als es Herr Dr. Schnabl durch die Aufstellung der Gattung Alloeostylus selbst gethan hat. Wir fragen daher, wie es um die Logik des Herrn Doctors bestellt sei ?

Gewiss wissen wir jede wissenschaftliche Arbeit $\mathrm{zu}$ schätzen, selbst wenn sie nicht fehlerfrei ist: dass man sich aber erkühnt, einer von den besten Intentionen geleiteten Kritik in ganz unbegründeter, verletzender Weise zu begegnen und dem Kritiker nicht nur unter Einem Fehler seiner Arbeiten unberechtigt vorzuwerfen, sondern seine ganze Thätigkeit auf entomologischen Gebiete herabzuw ürdigen, über sich bald darauf aber dieselbe Kritik ausübt und, wie ich zeigen

Wiener Entomologische Zeitung, VII. Jahrg., 4. Heft (30. April 1888). 
werde, jene Fehler selbst begeht, die man dem Kritiker zuschreibt; für diese Vorgänge finde Worte wer will.

Alloeostylus Schn. (olim) ist nach der l. c. gegebenen Beschreibung nichts anderes als Aricia s. str.; da diese Beschreibung mangelhaft war, konnte man verleitet werden, diese Gattung etwa auch zu Trichopticus Rond. zu stellen. Sie wurde einzig und allein auf ein dem Männchen zukommendes Merkmal, nämlich auf die Bildung der äusseren Genitalwerkzeuge aufgestellt. Welch geringen generischen Werth die Form des Hypopygs und seiner äusseren Anhänge bei den Anthomyiden besitzt, habe ich in der Wien. Ent. Ztg. 1887, pag. 271, zur Genüge dargethan. Wir werden dies weiter unten durch Herrn Dr. Schnabl selbst bestätigt finden. - Ein auf ein solches sexuelles Merkmal errichtetes Genus hat selbstverständlich keinen Werth.

In der That bringt nun Herr Dr. Schnabl in den Entom. Nachr. 1888, pag. 82 (Nachtrag zur Gatt. Alloeostylus Schn.) selbst die Correctur, da er auch Arten in seine Gattung Alloeostylus stellen muss, welche nicht jene Beschaffenheit des Hypopygs zeigen, wie die Art, auf welche er das Genus Alloeostylus anfänglich begründete, d. h. nichts anderes als: die Gattung Alloeostylus zeigt ein so veränderliches Hypopyg, wie seine Aricia s. lat.!

Doch da taucht der Autor mit den "Kreuzborsten“ auf und rettet in der That sein Genus Alloeostylus. Was geschieht aber mit seiner vielverfochtenen Aricia s. lat.? Die geht in die Brüche, wie ich es in meiner von Herrn Dr. Schnabl verpönten ursprünglichen Kritik vorausgesagt habe! Sowie die Beborstung der Mittelschenkel und alle die übrigen von Herrn Dr. Schnabl in den Entom. Nachr. 1888, pag. 8\% zur Rettung der Gattung Alloeostylus angeführten Thatsachen nur secundäre sexuelle Merkmale für das $\sigma$ sind, so sind es auch die Kreuzborsten für das $q$.

Während Herr Dr. Schnabl seiner Gattung All. durch secundäre sexuelle Merkmale dem männlichen und weiblichen Geschlechte nach Geltung zu verschaffen sucht, so will er ich muss es hier bemerken - meine aus Clinocera M. ausgeschiedenen Gattungen vom Schauplatz verschwinden machen, obgleich sie durchwegs auf Charaktere, welche beiden Geschlechtern gemeinsam zukommen, also nicht auf 
secundäre sexuelle Merkmale hin $€$ rkannt und errichtet worden sind (conf. Entom. Nachr. 1887, pag. 346).

Was nun Alloeostylus betrifft, so wird noch zu prüfen sein, ob diese Gattung wirklich von Aricia s. str. abzutrennen ist. Fest steht aber die Thatsache, dass sie von dem in den "Contributions" dargelegten Ansichten des Herrn Dr. Schn. über das "sogenannte" Genus Aricia sens. lat. absorbirt wird, so gut wie Herr Dr. Schn. die übrigen Genera Spilogaster, Trichpoticus etc. in diesen seinen verwitterten „Mischkrug" Aricia s. l. zu werfen beliebte.

Welche neuerliche Inconsequenz beging also der Herr Doctor mit Aufstellung seiner Gattung Alloeostylus! Die Verbissenheit in das Hypopygium war die einzige Ursache der Abtrennung derselben von der genannten Collectiv-Gattung. Welche Ausschreitungen ein solches Starrhalten an einer Ansicht bewirkt, sehen wir hier so deutlich, wie an den Exstir. pationen der Hypopygien getrockneter Mycetophiliden, und ich sprecbe hier nochmals mein Bedauern aus, dass zu solch praktisch schwer verwerthbaren Dingen eine wichtige Typensammlung wie die von Meister Winnertz dem theilweisen Untergang zugeführt wurde (vergl. Verh. Zool. Bot. Ges. 1886, pg. 480).

Ich wiederhole, dass alle die von Herrn Dr. Schnabl im Nachtrage zur Gattung Alloeostylus erbrachten Merkmale (Ent. Nachr. 1888, pag. 82) zum mindesten nicht mehr wiegen, als jene, auf welche man die Gattungen Aricia s. str., Spilogaster, Trichopticus etc. begründet hat, während Alloeostylus die wesentlichen Merkmale mit Aricia s. l. doch gemein hat. Ich will nur erwähnen, dass es sich zufolge der Untersuchungen des Herrn Dr. Schn. jetzt herausgestellt hat, dass einige Arten des Genus Aricia s. lat. sogar theilweise in der Kopfbildung mit Coenosia übereinstimmen: ein Vorkommen, wie es bei polysynthetischen Formen eben nicht anders zu erwarten ist. Das beweist aber noch nicht die generelle Wichtigkeit eines solchen Merkmales. Es fiel mir nicht ein, meine Chortophila rupicola, weil sie die Hinterkopfbildung (vgl. Wien. Ent. . Ztg. 1887, Taf. V, Fig. 10) mit Coenosia gemein hat, aus der Gattung Chortophila auszuscheiden.

Hält man Alloeostylus neben Aricia s. str., Spilogaster, Trichopticus etc. aufrecht, was wohl Herrn Dr. Schn. am liebsten sein wird, so ist jetzt schon die Vorhersagung in 
meiner ursprürglichen Kritik über die „Contributions“ in Erfüllung gegangen und hiermit auch die Berechtigung dieser ïbel aufgenommenen Kritik bewiesen. In derselben habe ich ausdrücklich gesagt: „Wir sind überzeugt, dass diejenigen Gattungen, welche Herr Dr. Schn. in sein grosses Genus Aricia s. l. gebracht, noch nicht gehörig systematisch auseinander gearbeitet sind (trotz der Contributions), und dass sich erst dann, wenn dies geschehen, zeigen wird, wie jene Arten, welche jetzt noch systematische Störenfriede bilden, sich ganz gut werden vertheilen lassen" (conf. Ent. Nachr. 1887, pg. 235). Herr Dr. Schn. selbst überzeugt uns von der Wahrheit dieses Ausspruches, da er A. Alaveola Fall. und A. simplex W., die gewiss solche Störenfriede waren, zur Ruhe bringt, indem er sie in das Genus Alloeostylus verweist (womit er freilich seine Inconsequenz leuchten lässt, da er neben Aricia s. l. seinen Alloeostylus aufrecht hält), und ähnlich wird es auch noch mit anderen Arten geschehen müssen, welche jetzt scheinbare Schwierigkeiten bei der Begrenzung der von Herrn Dr. Schn. in das Genus Aricia s. 1. eingezogenen, von Autoritäten längst anerkannten Gattungen bereiten.

Vielleicht kommt Herr Dr. Schn. aber mit dem Einwan de : die "Kreuzborsten“ sind jenes Moment, welches meine Argumente zu nichte macht. Sie kommen, wie er sagt, nur bei jenen Gattungen vor, welche er in den Ent. Nachr. 1888, pag. 82, mit einer solchen apodictischen Gewissheit namhaft gemacht hat, dass man über seine Kenntniss der Anthomyiden staunen muss (unterdessen dies Loew's Untersuchungen sind).

Allein, wenn Hydrophoria, Hydrotaea u. s. w. Kreuzborsten tragen, können nicht auch einzelne Arten im "Mischkruge" Aricia solche Borsten aufweisen? Wir haben ja erwähnt, dass die Anthomyiden polysynthetischer Natur sind und dass die genannten Borsten nur ein secundäres sexuelles Merkmal bedeuten, während Alloeostylus in beiden Geschlechtern jene wesentlichen Merkmale aufweist, wodurch diese Gattung in die Gattung Aricia s. lat. nothwendig gebracht werden musste und von Herrn Dr. Schn. thatsächlich auch gebracht wurde (A. Alaveola Fall. und A. simplex W.).

Nun noch einige Worte zur Aufklärung über die „Kreuzborsten". 
Dieser Terminus scheint mir ausser in Herrn Dr. Schn.'s "Nachtrag zur Gattung Alloeostylus" nur noch einmal in der gesammten dipterologischen Literatur angewendet worden zu sein. Möglicherweise, dass es öfters geschehen sei; sicher aber ist, dass diese Benennung von Loew herrührt. - Herr Dr. $\mathrm{Schnabl}$ hat, wie sich von selbst ergibt, zur Zeit der Abfassung seiner "Contributions" von diesem Merkmale und daher von diesem Ausdrucke noch nichts gewusst. Gewiss hätte er es sonst damals schon in seiner breitspurigen Charakteristik des Genus Aricia s. 1. erwähnt, da er ja in der genannten Arbeit Aricia aculeata Lw. (= A. Alavevla Fall.) zergliedert hat.

Es wäre nur recht und billig gewesen, wenn Herr Dr. Schnabl die Provenienz der Entdeckung der "Kreuzborsten" angegeben hätte. Dem Scharfsinne L o e w's haben wir die Entdeckung dieses Merkmales und die Benennung desselben zu verdanken. Der berühmte Autor wird freilich durch den Vorgang des Herrn Dr. Schn. nichts einbüssen; die Pietät allein aber, scheint mir, verlangt in dieser Richtung eine gewisse Rücksicht. Weit eher wäre es zu entschuldigen gewesen, wenn Herr Dr. Schn. bei der Taufe einer Lispe-Art das Andenken an Loew nicht so gewissenhaft bewahrt hätte, um sich hiermit selbst ein Denkmal zu setzen; ich meine Lispe Schnablii Schn. (Loew in litt.). Wenn auch die Literatur ähnliche einzelne Beispiele aufzuweisen hat, so sind sie keineswegs nachahmungswürdig.

Auch für mich möchte ich noch eine Lanze einlegen, was Herrn Dr. Schnabl erinnern mag, dass er Niemanden um sein Verdienst schmälern soll: bei Aufstellung der Synonymie von Aricia flaveola Fall. (vide Ent. Nachr. 1888, pag. 83) hat er es für gut befunden, alle anderen hierher bezüglichen Autoren zu nennen, meine Wenigkeit aber zu ignoriren, während ich doch der Erste war, der die ganze Synonymie dieser Art eigentlich festgestellt hat! (conf. Ent. Nachr. XII, 1886, pag. 217).

P. S. Ich bedauere recht sehr, dass vorstehender Artikel bereits abgedruckt war, als mir jener Aufsatz des H. Dr. Schn. (Entom. Nachricht. 1888, pg. 113) zu Gesichte kam, in welchem derselbe meiner Kritik der Contributions selbst Recht gibt. Da aber einer dem andern das „suum cuique“ nicht vorenthielt, glaube ich, unter der Versicherung auch meiner persönlichen Werthschätzung gegenüber H. Dr. Schn., dass die Sache jetzt zur Rahe gekommen sein wird. 


\section{$2 \mathrm{BHL}$ Biodiversity Heritage Library}

Mik, Josef. 1888. "Ueber die Dipterengattung Alloeostylus SCHNABL und über die sogenannten Kreuzborsten bei Anthomyiden-Weibchen." Wiener entomologische Zeitung 7, 135-139. https://doi.org/10.5962/bhl.part.27358.

View This Item Online: https://www.biodiversitylibrary.org/item/43819

DOI: https://doi.org/10.5962/bhl.part.27358

Permalink: https://www.biodiversitylibrary.org/partpdf/27358

\section{Holding Institution}

Smithsonian Libraries

\section{Sponsored by}

Smithsonian

\section{Copyright \& Reuse}

Copyright Status: NOT_IN_COPYRIGHT

This document was created from content at the Biodiversity Heritage Library, the world's largest open access digital library for biodiversity literature and archives. Visit BHL at https://www.biodiversitylibrary.org. 